\title{
Transcriptome profiling of anthocyanin-related genes reveals effects of light intensity on anthocyanin biosynthesis in red leaf lettuce
}

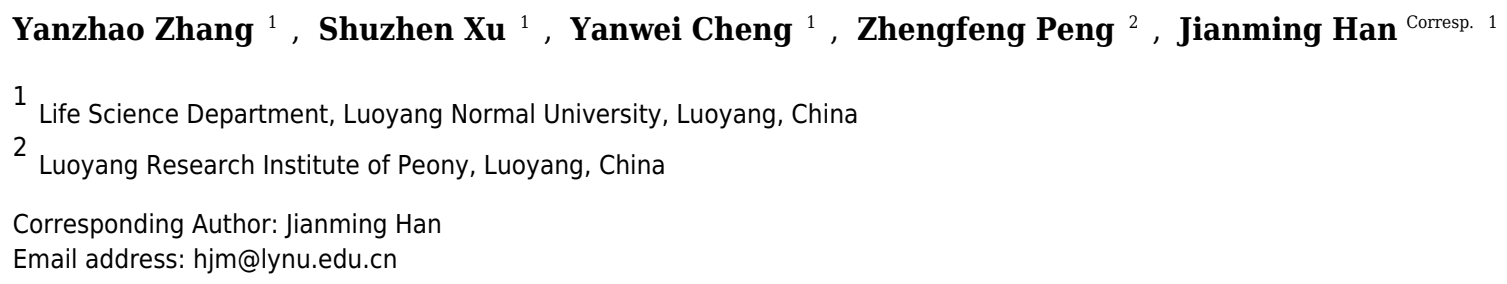

Red leaf lettuce (Lactuca sativa L.) is popular due to its high anthocyanin content, but poor leaf coloring often occurs under low light intensity. In order to reveal the mechanisms of anthocyanins affected by light intensity, we compared the transcriptome of Lactuca sativa L. var. capitata under light intensities of $40 \mu \mathrm{mol} \mathrm{m} \mathrm{m}^{-2} \mathrm{~s}^{-1}$ and $100 \mu \mathrm{mol} \mathrm{m} \mathrm{m}^{-2} \mathrm{~s}^{-1}$. A total of 62,111 unigenes were de novo assembled with an N50 of 1,681 bp, and 48,435 unigenes were functionally annotated in public databases. A total of 3,899 differentially expressed genes (DEGs) were detected, of which 1,377 unigenes were up-regulated and 2,552 unigenes were down-regulated in the high light samples. By KEGG enrichment analysis, the DEGs were significantly enriched in 14 pathways. Using gene annotation and phylogenetic analysis, we identified seven anthocyanin structural genes, including CHS, $C H I, F 3 H, F 3^{\prime} H, D F R, A N S$, and 3GT, and two anthocyanin transport genes, GST and MATE. In terms of anthocyanin regulatory genes, five MYBs and one bHLH gene were identified. An HY5 gene was discovered, which may respond to light signaling and regulate anthocyanin structural genes. These genes showed a log2FC of 2.7- 9.0 under high irradiance, and were validated using quantitative real-time (qRT)-PCR. In conclusion, our results indicated transcriptome variance in read leaf lettuce under low and high light intensity, and observed a anthocyanin biosynthesis and regulation pattern. The data should further help to unravel the molecular mechanisms of anthocyanins influenced by light intensity. 
1 Transcriptome profiling of anthocyanin-related genes reveals effects of light intensity on

2 anthocyanin biosynthesis in red leaf lettuce

3 Yanzhao Zhang ${ }^{1 \dagger}$, Shuzhen $\mathrm{Xu}^{1 \dagger}$, Yanwei Cheng ${ }^{1}$, Zhengfeng Peng ${ }^{2}$, Jianming Han ${ }^{1 *}$

$4 \quad{ }^{1}$ Life Science Department, Luoyang Normal University, Luoyang, China

$5 \quad{ }^{2}$ Luoyang Research Institute of Peony, Luoyang, China

6 †These authors contributed equally to this study

$7 \quad{ }^{*}$ Corresponding author: Jianming Han

8 E-mail: hjm@lynu.edu.cn

9

10

11

12

13

14

15

16

17

18

19

20

21

22

23

24

25 
28 Abstract.

29 Red leaf lettuce (Lactuca sativa L.) is popular due to its high anthocyanin content, but poor leaf coloring 30 often occurs under low light intensity. In order to reveal the mechanisms of anthocyanins affected by light intensity, we compared the transcriptome of Lactuca sativa L. var. capitata under light intensities of 40 $\mu \mathrm{mol} \mathrm{m} \mathrm{m}^{-2} \mathrm{~s}^{-1}$ and $100 \mu \mathrm{mol} \mathrm{m} \mathrm{m}^{-2} \mathrm{~s}^{-1}$. A total of 62,111 unigenes were de novo assembled with an N50 of $1,681 \mathrm{bp}$, and 48,435 unigenes were functionally annotated in public databases. A total of 3,899 differentially expressed genes (DEGs) were detected, of which 1,377 unigenes were up-regulated and 2,552 unigenes were down-regulated in the high light samples. By KEGG enrichment analysis, the DEGs were significantly enriched in 14 pathways. Using gene annotation and phylogenetic analysis, we identified seven anthocyanin structural genes, including $C H S, C H I, F 3 H, F 3$ ' $H, D F R, A N S$, and $3 G T$, and two anthocyanin transport genes, GST and MATE. In terms of anthocyanin regulatory genes, five MYBs and one bHLH gene were identified. An HY5 gene was discovered, which may respond to light signaling and regulate anthocyanin structural genes. These genes showed a $\log 2 \mathrm{FC}$ of 2.7- 9.0 under high irradiance, and were validated using quantitative real-time (qRT)-PCR. In conclusion, our results indicated transcriptome variance in read leaf lettuce under low and high light intensity, and observed a anthocyanin biosynthesis and regulation pattern. The data should further help to unravel the molecular mechanisms of anthocyanins influenced by light intensity.

\section{Introduction}

Color constitutes an important economic trait in vegetables and fruits, and can be mainly attributed to high anthocyanin accumulation. Anthocyanins are the primary pigments in plants and play an important role in the color development, generating a wide range of colors from pink to blue-purple. Anthocyanins have been verified to play a positive role in health, resulting in colorful foods gaining increasing global popularity (Espley et al., 2007; Chiu et al., 2010). The anthocyanin biosynthesis pathway is clear in plants, and three early biosynthesis genes (EBGs), including chalcone synthase (CHS),

52 chalcone isomerase (CHI), and

53 flavonoid 3-hydroxylase $(F 3 H)$, and four late biosynthesis genes (LBGs) flavonoid 3'-hydroxylase ( $F 3$ ' $H$ ), 54 dihydroflavonol reductase (DFR), anthocyanidin synthase (ANS) and 3-glucosyl transferase (3GT) have 55 been identified in the colored tissues of many plants. The enzymes encoded by these genes, in turn, 56 catalyze the substrate to synthesize anthocyanins (Winkel-Shirley, 2001). Anthocyanins are transferred to 57 the vacuoles by glutathione S-transferase and MATE-type proteins (Gomez et al., 2009;Sun et al., 2012), 
58 where they can function as bioactive molecules and dislay color. Anthocyanin pathways are mainly

59 regulated by genes from the MYB, bHLH, and WD40 families, and these proteins form a complex by

60 binding to the promoters of structural genes and regulating their transcription (Zhang et al.,

61 2003; Gonzalez et al., 2008;Xu et al., 2015). The MYB-bHLH-WD40 complex plays a central role in

62 regulating anthocyanins, and many abiotic stresses regulate anthocyanins mainly by activating or

63 inactivating the activity of this complex (Das et al., 2012;Zoratti et al., 2014).

64 Light is an important factor that influences anthocyanin accumulation in plants, and many

65 experiments have demonstrated that the transcription levels of anthocyanin regulatory and structural

66 genes decrease under light exclusion, which affects the anthocyanin content later on (Zoratti et al., 2014).

67 As observed in apple (Takos et al., 2006;Feng et al., 2013), Chinese bayberry (Niu et al., 2010), and pear

68 (Feng et al., 2010), the bagged fruit display no color, but upon exposure to sunlight, expression of the

69 anthocyanin related genes are up-regulated, leading to a red pericarp. R2R3 MYBs are important positive

70 regulators that directly influence the expression of the anthocyanin biosynthesis genes. Some R2R3

71 MYBs have been found to respond to light, such as MdMYB1 in apple (Takos et al., 2006), LrMYB15 in

72 Lilium regale (Yamagishi M., 2016), MrMYB1 in Chinese bayberry (Niu et al., 2010), LcMYB1 in litchi

73 (Lai et al., 2014), and VvMYBA1 and VvMYBA2 in grapevine (Azuma et al., 2012). Under changing

74 light conditions, the expression level of these R2R3 MYB transcription factors is adjusted to regulate the anthocyanin biosynthesis genes. ELONGATED HYPOCOTYL5 (HY5), a component of light-signaling pathways, has also been linked to the activation of the R2R3 MYBs and key anthocyanin biosynthesis genes in response to light in Arabidopsis and apple (Peng et al., 2013;Shin et al., 2013). Recent research revealed that light signals regulate anthocyanins via CONSTITUTIVE PHOTOMORPHOGENIC1 (COP1), which is a negative regulator and mediate degradation of anthocyanin positive regulators, like PAP1 and PAP2 in Arabiodpsis, MdMYB1 in apples (Li et al., 2012; Maier et al., 2013). Most light signal transduction experiments are analyzed by excluding light. However, Maier A and Hoecker U (2015) reported that the COP1/SPA complex may not be fully inactivated under low light intensity, suggesting the existence of some other light-intensity induced mechanism in anthocyanin accumulation.

Leaf color is an important factor influencing the consumer acceptance of red leaf lettuce, and light significantly influences the leaf color (Kang et al., 2013). In our previous work, we discovered that red leaf lettuce displayed a greener leaf color when grown under a light intensity of $40 \mu \mathrm{mol} \mathrm{m} \mathrm{m}^{-2} \mathrm{~s}^{-1}$, while an increase in the light intensity to $100 \mu \mathrm{mol} \mathrm{m} \mathrm{m}^{-2} \mathrm{~s}^{-1}$ was associated with a redder color. This red color was caused by increased anthocyanin accumulation. Zhang et al. (2016a) previously reported several putative genes involved in the anthocyanin biosynthetic pathway. However, the molecular mechanisms governing 90 light-induced anthocyanins in red leaf lettuce are still unknown. In this study, we mainly evaluated the 91 effect of light intensity on anthocyanin synthesis using comparative transcriptome analysis. The results 
92 should enhance our understanding of the correlation between light intensity and anthocyanin

93 accumulation at the molecular level.

94 Materials and Methods

95 Plant materials

96 Lactuca sativa L. var. "Capitata" was grown in the green house of the Luoyang Normal University 97 (China). Seedlings were grown under white light at an intensity $40 \mu \mathrm{mol} \mathrm{m} \mathrm{m}^{-2} \mathrm{~s}^{-1}$ and a $16 / 8 \mathrm{~h}$ day/night 98 photoperiod, with the temperature maintained at $22^{\circ} \mathrm{C} / 20^{\circ} \mathrm{C}$. Light intensity was measured using a FGH-1 99 photosynthetic radiometer (Beijing normal university photoelectric instrument factory). Forty days after 100 germination, one group of seedlings was supplied with a light intensity of $100 \mu \mathrm{mol} \mathrm{m}^{-2} \mathrm{~s}^{-1}$, and the other

101 group was used as a control. After three days of treatment, the two leaves at the top of plant were sampled, 102 and the leaves from five individuals were pooled in each group. Samples were frozen in liquid nitrogen 103 and stored at $-80^{\circ} \mathrm{C}$. Quantitative analysis of anthocyanin was performed as described by (Zhang et al., 104 2015), and cyanidin-3-O-glucoside (Cy3G) was used as standards for quantification. The soluble sugar 105 content was detected using the anthrone method ( $\mathrm{Li}, 2000)$.

106

107

108

109

110

111

112

113

114

115

116

117

118

119

120

121

cDNA library construction and sequencing

Total RNA was extracted using CTAB-LiCl method (Gambino et al., 2008), and genomic DNA contamination was removed using DNase I (TaKaRa, Japan). RNA quality was verified by agarose gel electrophoresis and a Bioanalyzer 2100 (Agilent, CA, USA). For cDNA library construction, mRNA was enriched with oligo (dT) magnetic beads and then broken into smaller pieces using fragmentation buffer. The first strand cDNA was reversed-transcribed by random hexamers and small fragment as templates. This was followed by second strand cDNA synthesis using DNA Polymerase I and RNase H. The double strand cDNA was ligated to paired-end adapters, and suitable fragments were selected and enriched with PCR amplification. cDNA libraries were sequenced performed using an Illumina HiSeq2000 platform at BGI Co., Ltd. Sequence data were deposited in the NCBI database with accession numbers SRR5868088 and SRR5943715.

\section{De novo assembly and gene annotation}

Raw reads were filtered by discarding the following: reads with adaptors, reads with unknown nucleotides larger than $5 \%$, and low quality reads (more than $10 \%$ of bases with a $\mathrm{Q}$ score $\leq 20$ ). The remaining clean reads from each sample were assembled using Trinity v2.0.6 software (Grabherr et al., 2011). TGICL v2.06 was then used to construct a non-redundant unigene set from the two assembled 
122 datasets (Pertea et al., 2003). Unigenes were aligned to functional databases to obtain gene functions,

123 mainly including the NCBI non-redundant (Nr), nucleotide ( $\mathrm{Nt})$, InterPro, SWISS-PROT, and Kyoto

124 Encyclopedia of Genes and Genomes (KEGG) databases by BLAST v2.2.23 with an E-value threshold

$125<1 \mathrm{e}-5$. Based on the Nr annotation, Gene Ontology (GO) annotation (http://www. geneontology.org) was

126 performed using the BLAST2GO program v2.5.0 (Conesa et al., 2005).

\section{Analysis of differentially expressed genes (DEGs)}

128 Clean reads were mapped to unigenes using Bowtie2 v2.2.5 (Langmead and Salzberg, 2012), and 129 gene expression level was calculated based on Fragments Per kb per Million fragments (FPKM) method 130 in RSEM v1.2.12 (Mortazavi et al., 2008). Significantly differentially expressed genes were scanned 131 among samples under low and high light using EBSeq package v1.7.1 (Leng et al., 2013), with a 132 threshold of an absolute $\log 2$ ratio $\geq 2$ and a false discovery rate (FDR) significance score $<0.001$. Based 133 on the KEGG and GO annotation, we classified DEGs and performed functional enrichment using phyper 134 within R. The terms in which FDR not larger than 0.001 are defined as significant enrichment.

\section{Prediction and sequence analysis of transcription factor}

136 ORF of each unigene was predicted using getorf EMBOSS:6.5.7.0 (Rice et al., 2000), and 137 hmmsearch v3.0 was adopted to identify transcription factor by aligning ORF to domain from PlantTFDB 138 (Mistry et al., 2013). Neighbour-joining phylogenetic analysis was carried out using MEGA5 (Tamura et 139 al., 2011).

\section{Quantitative-PCR Analysis}

141 Extraction of total RNA and elimination of genomic DNA contamination was performed as above, 142 and $2 \mu \mathrm{g}$ of total RNA was used as template and the first strand cDNA was synthesized using the 143 PrimeScript ${ }^{\mathrm{TM}} \mathbb{I}$ 1st strand cDNA Synthesis Kit (TAKARA, DaLian) with primer oligo (dT), according 144 to the manufacturer's instructions. Following 10 times dilution, the cDNA was used for quantitative 145 (q)PCR. qPCR was performed using the SYBR ${ }^{\circledR}$ Premix Ex Taq ${ }^{\mathrm{TM}}$ II kit (Tli RNaseH Plus; TAKARA, 146 DaLian) according to the manufacturer's instructions. Gene specific primers are shown in Table S1. The 147 thermal-cycling conditions were as follows: an initial heat denaturing step at $95^{\circ} \mathrm{C}$ for 3 min; then 40 148 cycles of $95^{\circ} \mathrm{C}$ for $10 \mathrm{~s}, 55^{\circ} \mathrm{C}$ for $20 \mathrm{~s}$, and $72^{\circ} \mathrm{C}$ for $20 \mathrm{~s}$. Each sample was amplified in three independent 149 replicates. Gene transcription levels were calculated using the $2^{-\Delta \Delta \mathbf{C T}}$ comparative threshold cycle $(\mathrm{Ct})$ 150 method (Livak and Schmittgen, 2001), and actin was used as an internal control to normalize the relative 151 expression levels of the analyzed genes (Borowski et al., 2014). 
152 Results

153 Light intensity determines anthocyanin content

154 After $3 \mathrm{~d}$ irradiation at a high light intensity of $100 \mu \mathrm{mol} \mathrm{m}^{-2} \mathrm{~s}^{-1}$, the plant turned red, while the plant 155 grown at low light intensity of $40 \mu \mathrm{mol} \mathrm{m}^{-2} \mathrm{~s}^{-1}$ still exhibited a pale green color (Figure 1A,B). High156 performance Liquid Chromatography (HPLC) analysis revealed that anthocyanins accumulated in the 157 plants under high light at a level of $2.1 \mathrm{mg} / \mathrm{g}$, but were barely detected in those grown under low light 158 (Figure 1C). Our results indicated that anthocyanins were induced as light intensity increased to $100 \mu \mathrm{mol}$ $159 \mathrm{~m}^{-2} \mathrm{~s}^{-1}$, and which resulted in the red coloring in leaves of lettuce. Soluble sugar content was also 160 increased with increasing light intensity (Figure 1D). The content increased 2.9-fold when irradiated 161 under $100 \mu \mathrm{mol} \mathrm{m} \mathrm{m}^{-2} \mathrm{~s}^{-1}$ in comparison to $40 \mu \mathrm{mol} \mathrm{m} \mathrm{m}^{-2} \mathrm{~s}^{-1}$.

\section{Transcriptome sequencing and de novo assembly}

163 After filtering raw data, we generated 59,774,792 clean reads, which included 8,966,218,800 nt from 164 samples under light intensity of $100 \mu \mathrm{mol} \mathrm{m}^{-2} \mathrm{~s}^{-1}$, and 58,856,106 clean reads that included 8,828,415,900 $165 \mathrm{nt}$ from samples under light intensity of $100 \mu \mathrm{mol} \mathrm{m} \mathrm{m}^{-2} \mathrm{~s}^{-1}$. Trinity software was used to perform de novo 166 assembly with the clean reads, generating 69,733 transcripts with an N50 of 1,500 bp in the high light

167 sample, and 79,037 transcripts in the low light sample with an N50 of 1,460 bp. Redundancy in the

168 transcripts was removed using Tgicl, finally resulting in the generation of 62,111 unigenes with an N50 of $1691,681 \mathrm{bp}$.

170 A total of 24,129 unigene sequences (38.8\%) had a length between 200 and 500 nucleotides (nt), 13,180

171 unigenes (21.2\%) were between 500 and $1000 \mathrm{nt}$ in length, 16,058 unigenes (25.8\%) were between 1,000

172 and 2,000 nt in length, and 8,744 unigenes (14.1\%) were longer than 2,000 nt.

173 After assembly, gene functions were predicted by querying seven public databases, and a total of 174 48,435 unigenes (77.98\%) were functionally annotated. Among them, 45,046 unigenes (72.52\%) obtained 175 hits in the Nr database, 31,786 obtained hits in the SWISS-PROT database, and 33,713 unigenes obtained 176 hits in the InterPro database. TransDecoder v3.0.1 (https://transdecoder.github.io) was used to predict the 177 ORFs. Overall, 22,623 genes were predicted to contain full-length ORFs, accounting for 36.42\% of the 178 assembled unigenes. Based on the $\mathrm{Nr}$ annotation, the top sequence matches obtained from BLASTX are 179 shown in Figure S1. The red leaf lettuce sequences had the highest similarity to Cynara cardunculus var. 180 scolymus sequences (57.02\%), followed by Vitis vinifera (5.29\%), Sesamum indicum (2.53\%), Coffea 181 canephora (1.95\%), and Nicotiana sylvestris (1.57\%).

\section{Differentially expressed genes (DEGs)}


A total of 3,929 unigenes were recovered as differentially expressed between samples grown under

184 low and high light conditions. Compared with the low light sample, 1,377 unigenes were up-regulated and 2,552 unigenes were down-regulated in the high light sample (Figure 2). To further understand the biological functions of the DEGs, we performed pathway analysis based on the KEGG database. A total of 2,891 unigenes were assigned to six categories including 132 KEGG pathways. KEGG pathway enrichment analysis was performed based on hypergeometric tests. The DEGs between the two samples were significantly enriched in 14 pathways (Figure 3). Among them, four pathways including "phenylpropanoid biosynthesis (ko00940)", "flavonoid biosynthesis (ko00941)", "flavone and flavonol biosynthesis (ko00944)", and "anthocyanin biosynthesis (ko00942)" are closely associated with anthocyanin biosynthesis, and three glucide metabolic pathways including "other glycan degradation (ko00511)", "starch and sucrose metabolism (ko00500)", and "galactose metabolism (ko00052)" may be related to the synthesis of substrates.

We assigned 1,257 of the 3,929 DEGs to three main GO categories including "molecular functions," "biological processes," and "cellular components" (Figure S2). Among them, 742 unigenes were grouped in the category "cellular components," 992 unigenes in "molecular function," and 884 unigenes in "biological processes." Under the GO category "molecular functions", the "polynucleotide adenylyltransferase activity" and "oxidoreductase activity" were the most highly enriched terms. Under the category "Molecular function", the "flavonoid biosynthetic process" were the most highly enriched term.

\section{Analysis of genes involved in anthocyanin biosynthesis and transport}

Using gene annotation and phylogenetic analysis, we identified putative structural genes involved in anthocyanin synthesis and transport (Table 1). The accession numbers in Genbank were MF579543MF579560. In terms of anthocyanin structural genes, a total of nine genes covered each step of the anthocyanin biosynthetic pathway. Among them, the CHS and $3 G T$ gene family contained two members, while the other genes contained only one member. All nine candidate genes were up-regulated under high light, and their normalized transcript levels were 2- to 9-fold higher than under low light. Among the CHS and 3GT gene family members, Unigene12000_All and CL4808.Contig1_All displayed greater transcript abundance and more obvious growth at the transcription level. It is indicated that Unigene12000_All and CL4808.Contig1_All may be more insensitive to light intensity and contribute more to anthocyanin biosynthesis. In brief, all the putative structural genes were co-up-regulated and the anthocyanin pathway was active under high light conditions.

We also detected the differential expression of two anthocyanin transport genes, GST (Unigene10814_All) and MATE (Unigene12020_All), with transcript levels 7.1- and 1.1-fold higher 
216 under high light conditions. GST possessed higher transcript abundance and was significantly up-

217 regulated, suggesting that anthocyanins might be primarily transported by GST.

\section{Transcription factors regulating anthocyanin biosynthesis}

219 We predicted a total of 291 transcription factors (TFs) belonging to 39 families (Table S2). The 220 MYB gene family represented the largest group containing 33 members, followed by the AP2-EREBP 221 (30 members), MYB-related (26 members), and bHLH gene families (19 members). In model plants, TFs 222 from the MYB, bHLH, and WD40 families regulate transcripts of anthocyanin structural genes. In the 33 223 MYBs filtered from the detected DEGs, 14 were up-regulated and 19 were down-regulated under high 224 light conditions. Among the up-regulated genes, Unigene12430_All, Unigene12294_All, and 225 Unigene23058_All formed a clade with anthocyanin regulatory genes from Arabidopsis, grape, and 226 Antirrhinum, which have been shown to play a central role in regulating LBGs. Unigene24751_All and 227 CL6440.Contig1_All were closely associated with MYB12 of Arabidopsis, which mainly regulates EBGs 228 (Figure 4a). Following treatment with high light, the transcription levels of MYBs were up-regulated 1.9229 to 6.0 -fold.

230 In our study, six bHLH TFs were up-regulated and 13 were down-regulated under high light 231 conditions. Of these, Unigene13011_All was predicted as a anthocyanin regulatory gene and was up232 regulated 2.6-fold under high light. Sequence alignment revealed that it contained the BOX18, BOX19, 233 and HLH motifs, which were depicted as conserved in sub group III of the bHLH gene family. It grouped 234 with bHLH in the phylogeny (Figure 4b), which has been proven to regulated anthocyanin in apple, 235 Petunia, and Arabidopsis, and was most closely related to DvIVS with 62.6\% amino acid similarity.

236 HY5 is a member of the bZIP gene family and acts downstream of the light receptor network and 237 directly affects the transcription of light-induced genes. It was previously verified to regulate anthocyanin 238 structural genes and PAP1 expression by directly binding to their promoters (Shin et al., 2007;Shin et al., 239 2013). In the bZIP family, 14 genes were up-regulated and 19 were down-regulated. Phylogenetic 240 analysis placed Unigene19629_All in the same cluster as the HY5 proteins from different plant species 241 (Figure S3). Unigene19629_All showed 65.5\% amino acid similarity with AtHY5 in Arabidopsis, the 242 transcription level of which was up-regulated 1.7-fold under high irradiance.

\section{Quantitative real-time (qRT)-PCR validation of DEGs}

244 To further validate the comparative transcriptome results, the transcript level variances of the 18 245 putative genes involved in anthocyanin synthesis, transport, and regulation between the low and high light 246 conditions were detecting using qRT-PCR analysis. The results indicated that the transcript levels of 17 
247 genes were significantly up-regulated in the high light samples (Figure 5), which is in agreement with the

248 alterations in gene expression detected by the transcriptome analysis. However, the MYB gene

249 CL6440.Contig1_All was not significantly up regulated under light intensity of $100 \mu \mathrm{mol} \mathrm{m}^{-2} \mathrm{~s}^{-1}$. Overall,

250 these results indicate that the transcriptomic profiling data correlate with the light intensity responses of

251 red leaf lettuce.

\section{Discussion}

253 Light is one of the most important environmental factors affecting anthocyanin biosynthesis in plants.

254 Generally, high light intensity is required for the induction of anthocyanin synthesis, and under different

255 light exposure levels, anthocyanin contents have been found to vary in plants and even individual leaves.

256 As found in Lisianthus, the flowers exhibited a paler color under low light conditions, with a 30\%

257 reduction in anthocyanin content and $40 \%$ reduction in color intensity associated with a $25 \%$ decrease

258 from sunlight (Griesbach, 1992). In this study, the leaves of "Capitata" were green under $40 \mu \mathrm{mol} \mathrm{m}^{-2} \mathrm{~s}^{-1}$,

259 but displayed a red pigment when treated with a higher light intensity of $100 \mu \mathrm{mol} \mathrm{m} \mathrm{m}^{-2} \mathrm{~s}^{-1}$. Our results

260 demonstrated that a certain level of light intensity is necessary for anthocyanin accumulation in red leaf

261 lettuce, which is consistent with previous findings of color variance in lettuce (Voipio and Autio, 1995).

262 The MYB-bHLH-WD40 complex plays a central role in regulating the anthocyanin pathway, and the

263 environmental factors that affect anthocyanin content usually control the transcript levels of the regulated

264 genes. Many studies have revealed that light mainly affects anthocyanin content via regulation of the

265 transcription levels of MYB-regulated genes, including in apple (Takos et al., 2006), Chinese bayberry

266 (Niu et al., 2010), pear (Feng et al., 2010) and grapevine (Azuma et al., 2012), and the transcription levels

267 of these MYBs change dynamically in response to the light conditions, which is associated with variation

268 in color. In many plants, multiple MYB genes have been shown to redundantly regulate the anthocyanin

269 biosynthesis pathway. For example, in Arabidopsis, PAP1 and PAP2 regulate the late biosynthetic genes,

270 while MYB11, MYB12, and MYB111 mainly regulate the early biosynthetic genes (Stracke et al., 2007).

271 Recently, bHLH genes were reported to be significantly up-regulated when exposed to higher light

272 intensity in Chrysanthemum (Hong et al., 2015), the pericarps of litchi (Zhang et al., 2016b), and peach

273 (Liu et al., 2015). We identified four MYBs and one bHLH gene that were up-regulated under high light

274 conditions, as well as structural genes. Our results indicated that higher light intensity up-regulated the

275 transcript levels of these five genes, and further activated the anthocyanin pathway. MYB1 mainly

276 regulates the late biosynthetic genes, while MYB11, MYB12, and MYB111 mainly regulate the early

277 biosynthetic genes. For the MYB gene CL6440.Contig1_All, a low transcript abundance may explain the 
278 variance in transcriptional level obtained by the transcriptome sequencing and qPCR, and it may not be

279 primarily responsible for the regulation of the early anthocyanin pathway.

280 A ubiquitin E3 ligase CONSTITUTIVE PHOTOMORPHOGENIC1 (COP1) was recently found to 281 repress the activity of positive regulators of anthocyanins at the post-translational level (Li et al.,

282 2012;Maier et al., 2013). In darkness, the COP1/SUPPRESSOR OF PHYA (SPA) complex localizes to

283 the nucleus where it interacts with the positive regulators of anthocyanins, mediating their ubiquitination

284 and degradation via the $26 \mathrm{~S}$ proteasome pathway. Conversely, under high light conditions, COP1

285 dissociates from the COP1/SPA complex via the activated photoreceptors, and is exported from the

286 nucleus. The low COP1 abundance in the nucleus then allows nuclear-localized transcription factors to

287 accumulate and induce gene expression (Lau and Deng, 2012). Recently research also showed that light

288 affects anthocyanin biosynthesis via transcriptional regulation of COP1. Like in litch (Zhang et al.,

289 2016b), crabapple (Lu et al., 2016), and eggplant (Jiang et al., 2016), transcript levels of COPI was

290 decreased from dark to sunlight exposed condition. In our study, the increased light intensity from 40 to

$291100 \mu \mathrm{mol} \mathrm{m}{ }^{-2} \mathrm{~s}^{-1}$ activated the anthocyanin pathway, but the transcript levels of the COP1 gene were not

292 significantly elevated. We suggested that transcript level of COPI was not sensitive to variation of light

293 intensity in red leaf lettuce, and the the activity of anthocyanin pathway may related with COP1

294 subcellular localization.

295 Sugars are endogenous triggers that modulate the expression of anthocyanin biosynthetic genes by

296 acting as signaling molecules and activating anthocyanin regulatory genes by means of a sucrose-specific

297 signaling pathway (Solfanelli et al., 2006). Increasing sucrose concentrations usually induce anthocyanin

298 accumulation, which has even been proposed as a useful phenotypic marker for soluble carbohydrate

299 accumulation (Hu et al., 2002). Several studies suggest that low light intensity affects anthocyanin

300 accumulation through reduced photosynthesis in the leaves or stems, which, in turn, reduces the soluble

301 sugar content of petals and leads to a repression of the genes that encode enzymes of the anthocyanin

302 biosynthetic pathway (Kawabata et al., 1995;Meir et al., 2010). Our KEGG enrichment analysis revealed

303 four sugar pathways and four anthocyanin-related pathways, which indicated that a higher light intensity

304 of $100 \mu \mathrm{mol} \mathrm{m} \mathrm{m}^{-2} \mathrm{~s}^{-1}$ increased soluble sugar concentration during photosynthesis, which not only provides

305 a substrate for anthocyanins, but also activates signals to regulate the expression of anthocyanin-related 306 genes.

\section{Conclusions}


308 In red leaf lettuce, anthocyanin content is regulated by light intensity. Under low light conditions (40

$309 \mu \mathrm{mol} \mathrm{m} \mathrm{m}^{-2} \mathrm{~s}^{-1}$ ), the anthocyanin pathway is inactive. When the light intensity is increased to $100 \mu \mathrm{mol} \mathrm{m} \mathrm{m}^{-2} \mathrm{~s}^{-}$

$310^{1}$, the putative genes corresponding to anthocyanin biosynthesis, transport, and regulation were

311 significantly up-regulated. Glucide metabolic may play important role in anthocyanin accumulation as the

312 increased light intensity.

313 Acknowledgements

314 We thank Huiping Ma of Luoyang Research Institute of Peony (Luoyang, China)

315 for providing advice for experiments.

\section{References}

Azuma, A., Yakushiji, H., Koshita, Y., and Kobayashi, S. (2012). Flavonoid biosynthesis-related genes in grape skin are differentially regulated by temperature and light conditions. Planta 236, 1067-1080. doi: 10.1007/s00425-012-1650-x.

Borowski, J.M., Galli, V., Messias Rda, S., Perin, E.C., Buss, J.H., Dos Anjos E Silva, S.D., and Rombaldi, C.V. (2014). Selection of candidate reference genes for real-time PCR studies in lettuce under abiotic stresses. Planta 239, 11871200. doi: 10.1007/s00425-014-2041-2.

Chiu, L.-W., Zhou, X., Burke, S., Wu, X., Prior, R.L., and Li, L. (2010). The Purple Cauliflower Arises from Activation of a MYB Transcription Factor. Plant Physiol. 154, 1470. doi: https://doi.org/10.1104/pp.110.164160.

Conesa, A., Gotz, S., Garcia-Gomez, J.M., Terol, J., Talon, M., and Robles, M. (2005). Blast2GO: a universal tool for annotation, visualization and analysis in functional genomics research. Bioinformatics 21, 3674-3676. doi: 10.1093/bioinformatics/bti610.

Das, P.K., Shin, D.H., Choi, S.B., and Park, Y.I. (2012). Sugar-hormone cross-talk in anthocyanin biosynthesis. Mol. Cells 34, 501-507. doi: 10.1007/s10059-012-0151-x.

Espley, R.V., Hellens, R.P., Putterill, J., Stevenson, D.E., Kutty-Amma, S., and Allan, A.C. (2007). Red colouration in apple fruit is due to the activity of the MYB transcription factor, MdMYB10. Plant J. 49, 414-427. doi: 10.1111/j.1365313X.2006.02964.X.

Feng, F., Li, M., Ma, F., and Cheng, L. (2013). Phenylpropanoid metabolites and expression of key genes involved in anthocyanin biosynthesis in the shaded peel of apple fruit in response to sun exposure. Plant Physiol. Biochem. 69, 5461. doi: 10.1016/j.plaphy.2013.04.020.

Feng, S., Wang, Y., Yang, S., Xu, Y., and Chen, X. (2010). Anthocyanin biosynthesis in pears is regulated by a R2R3-MYB transcription factor PyMYB10. Planta 232, 245-255. doi: 10.1007/s00425-010-1170-5.

Gambino, G., Perrone, I., and Gribaudo, I. (2008). A Rapid and effective method for RNA extraction from different tissues of grapevine and other woody plants. Phytochem Anal 19, 520-525. doi: 10.1002/pca.1078.

Gomez, C., Terrier, N., Torregrosa, L., Vialet, S., Fournier-Level, A., Verriès, C., Souquet, J.-M., Mazauric, J.-P., Klein, M., Cheynier, V., and Ageorges, A. (2009). Grapevine MATE-Type Proteins Act as Vacuolar $\mathrm{H}^{+}$-Dependent Acylated Anthocyanin Transporters. Plant Physiology 150, 402-415. doi: 10.1104/pp.109.135624.

Gonzalez, A., Zhao, M., Leavitt, J.M., and Lloyd, A.M. (2008). Regulation of the anthocyanin biosynthetic pathway by the TTG1/bHLH/Myb transcriptional complex in Arabidopsis seedlings. Plant J. 53, 814-827. doi: 10.1111/j.1365313X.2007.03373.X.

Grabherr, M.G., Haas, B.J., Yassour, M., Levin, J.Z., Thompson, D.A., Amit, I., Adiconis, X., Fan, L., Raychowdhury, R., Zeng, Q., Chen, Z., Mauceli, E., Hacohen, N., Gnirke, A., Rhind, N., Di Palma, F., Birren, B.W., Nusbaum, C., Lindblad-Toh, K., Friedman, N., and Regev, A. (2011). Full-length transcriptome assembly from RNA-Seq data without a reference genome. Nat. Biotechnol. 29, 644-652. doi: 10.1038/nbt.1883.

Griesbach, R.J. (1992). Correlation of pH and Light Intensity on Flower Color in Potted Eustoma grandiflorum Grise. HortScience 27, 817-818.

Hong, Y., Tang, X., Huang, H., Zhang, Y., and Dai, S. (2015). Transcriptomic analyses reveal species-specific light-induced anthocyanin biosynthesis in chrysanthemum. BMC Genomics 16, 202. doi: 10.1186/s12864-015-1428-1.

$\mathrm{Hu}$, Z., Larson, S.R., Jones, T., and Wang, R.R.C. (2002). Genetic relationships among soluble carbohydrates, anthocyanins and growth characteristics in Leymus (Gramineae) detected with molecular markers. 
355

Jiang, M., Ren, L., Lian, H., Liu, Y., and Chen, H. (2016). Novel insight into the mechanism underlying light-controlled anthocyanin accumulation in eggplant (Solanum melongena L.). Plant Sci 249, 46-58. doi: 10.1016/j.plantsci.2016.04.001.

Kang, J.H., Krishnakumar, S., Atulba, S.L.S., Jeong, B.R., and Hwang, S.J. (2013). Light intensity and photoperiod influence the growth and development of hydroponically grown leaf lettuce in a closed-type plant factory system. Hortic., Environ. Biotechnol. 54, 501-509. doi: 10.1007/s13580-013-0109-8.

Kawabata, S., Ohta, M., Kusuhara, Y., and Sakiyama, R. (1995). Influences of low light intensities on the pigmentation of eustoma grandiflorum flowers. Acta Hortic. , 173-178. doi: 10.17660/ActaHortic.1995.405.22.

Lai, B., Li, X.J., Hu, B., Qin, Y.H., Huang, X.M., Wang, H.C., and Hu, G.B. (2014). LcMYB1 is a key determinant of differential anthocyanin accumulation among genotypes, tissues, developmental phases and ABA and light stimuli in Litchi chinensis. PLoS One 9, e86293. doi: 10.1371/journal.pone.0086293.

Langmead, B., and Salzberg, S.L. (2012). Fast gapped-read alignment with Bowtie 2. Nat. Methods 9, 357-359. doi: 10.1038/nmeth.1923.

Lau, O.S., and Deng, X.W. (2012). The photomorphogenic repressors COP1 and DET1: 20 years later. Trends Plant Sci. 17, 584593. doi: 10.1016/j.tplants.2012.05.004.

Leng, N., Dawson, J.A., Thomson, J.A., Ruotti, V., Rissman, A.I., Smits, B.M., Haag, J.D., Gould, M.N., Stewart, R.M., and Kendziorski, C. (2013). EBSeq: an empirical Bayes hierarchical model for inference in RNA-seq experiments. Bioinformatics 29, 1035-1043. doi: 10.1093/bioinformatics/btt087.

Li Hesheng. Plant physiology and biochemistry experiment principle and technology[M].Beijing: Higher education press.2003:156-165.

Li, Y.Y., Mao, K., Zhao, C., Zhao, X.Y., Zhang, H.L., Shu, H.R., and Hao, Y.J. (2012). MdCOP1 ubiquitin E3 ligases interact with MdMYB1 to regulate light-induced anthocyanin biosynthesis and red fruit coloration in apple. Plant Physiol. 160, 1011-1022. doi: 10.1104/pp.112.199703.

Liu, T., Song, S., Yuan, Y., Wu, D., Chen, M., Sun, Q., Zhang, B., Xu, C., and Chen, K. (2015). Improved peach peel color development by fruit bagging. Enhanced expression of anthocyanin biosynthetic and regulatory genes using white nonwoven polypropylene as replacement for yellow paper. Sci. Hortic. 184, 142-148. doi: http://dx.doi.org/10.1016/j.scienta.2015.01.003.

Livak, K.J., and Schmittgen, T.D. (2001). Analysis of Relative Gene Expression Data Using Real-Time Quantitative PCR and the 2- $\Delta \Delta$ CT Method. Methods 25, 402-408. doi: http://dx.doi.org/10.1006/meth.2001.1262.

Lu, Y., Hao, S., Liu, N., Bu, Y., Yang, S and Yao Y. (2016) Light affects anthocyanin biosynthesis via transcriptional regulation of COP1 in the ever-red leaves of crabapple M.cv. 'Royalty'. Braz. J. Bot 39: 659. https://doi.org/10.1007/s40415-0160277-8.

Maier, A., and Hoecker, U. (2015). COP1/SPA ubiquitin ligase complexes repress anthocyanin accumulation under low light and high light conditions. Plant Signal. Behav. 10, e970440. doi: 10.4161/15592316.2014.970440.

Maier, A., Schrader, A., Kokkelink, L., Falke, C., Welter, B., Iniesto, E., Rubio, V., Uhrig, J.F., Hulskamp, M., and Hoecker, U. (2013). Light and the E3 ubiquitin ligase COP1/SPA control the protein stability of the MYB transcription factors PAP1 and PAP2 involved in anthocyanin accumulation in Arabidopsis. Plant J. 74, 638-651. doi: 10.1111/tpj.12153.

Meir, S., Kochanek, B., Glick, A., Salim, S., Lers, A., Burd, S., Philosoph-Hadas, S., and Weiss, D. (2010). Reduced petal pigmentation in lisianthus (eustoma grandiflorum) flowers under low light conditions is associated with decreased expression of anthocyanin biosynthesis genes. Acta Hortic. 1735-1744. doi: 10.17660/ActaHortic.2010.877.238.

Mistry, J., Finn, R.D., Eddy, S.R., Bateman, A., and Punta, M. (2013). Challenges in homology search: HMMER3 and convergent evolution of coiled-coil regions. Nucleic Acids Res. 41, e121. doi: 10.1093/nar/gkt263.

Mortazavi, A., Williams, B.A., Mccue, K., Schaeffer, L., and Wold, B. (2008). Mapping and quantifying mammalian transcriptomes by RNA-Seq. Nat. Methods 5, 621-628. doi: 10.1038/nmeth.1226.

Niu, S.S., Xu, C.J., Zhang, W.S., Zhang, B., Li, X., Lin-Wang, K., Ferguson, I.B., Allan, A.C., and Chen, K.S. (2010). Coordinated regulation of anthocyanin biosynthesis in Chinese bayberry (Myrica rubra) fruit by a R2R3 MYB transcription factor. Planta 231, 887-899. doi: 10.1007/s00425-009-1095-z.

Peng, T., Saito, T., Honda, C., Ban, Y., Kondo, S., Liu, J.-H., Hatsuyama, Y., and Moriguchi, T. (2013). Screening of UV-Binduced genes from apple peels by SSH: possible involvement of MdCOP1-mediated signaling cascade genes in anthocyanin accumulation. Physiologia Plantarum 148, 432-444. doi: 10.1111/ppl.12002.

Pertea, G., Huang, X., Liang, F., Antonescu, V., Sultana, R., Karamycheva, S., Lee, Y., White, J., Cheung, F., Parvizi, B., Tsai, J., and Quackenbush, J. (2003). TIGR Gene Indices clustering tools (TGICL): a software system for fast clustering of large EST datasets. Bioinformatics 19, 651-652. doi: https://doi.org/10.1093/bioinformatics/btg034.

Rice, P., Longden, I., and Bleasby, A. (2000). EMBOSS: the European Molecular Biology Open Software Suite. Trends Genet. 16, 276-277. doi: 10.1002/0471650129.dob0206.

Shin, D.H., Choi, M., Kim, K., Bang, G., Cho, M., Choi, S.B., Choi, G., and Park, Y.I. (2013). HY5 regulates anthocyanin biosynthesis by inducing the transcriptional activation of the MYB75/PAP1 transcription factor in Arabidopsis. FEBS Lett. 587, 1543-1547. doi: 10.1016/j.febslet.2013.03.037.

Shin, J., Park, E., and Choi, G. (2007). PIF3 regulates anthocyanin biosynthesis in an HY5-dependent manner with both factors directly binding anthocyanin biosynthetic gene promoters in Arabidopsis. Plant J. 49, 981-994. doi: 10.1111/j.1365313X.2006.03021.x. 
416

417

418

419

420

421

422

423

424

425

426
Solfanelli, C., Poggi, A., Loreti, E., Alpi, A., and Perata, P. (2006). Sucrose-specific induction of the anthocyanin biosynthetic pathway in Arabidopsis. Plant Physiol. 140, 637-646. doi: 10.1104/pp.105.072579.

Stracke, R., Ishihara, H., Huep, G., Barsch, A., Mehrtens, F., Niehaus, K., and Weisshaar, B. (2007). Differential regulation of closely related R2R3-MYB transcription factors controls flavonol accumulation in different parts of the Arabidopsis thaliana seedling. Plant J. 50, 660-677. doi: 10.1111/j.1365-313X.2007.03078.x.

Sun, Y., Li, H., and Huang, J.-R. (2012). Arabidopsis TT19 Functions as a Carrier to Transport Anthocyanin from the Cytosol to Tonoplasts. Molecular Plant 5, 387-400. doi: https://doi.org/10.1093/mp/ssr110.

Takos, A.M., Jaffe, F.W., Jacob, S.R., Bogs, J., Robinson, S.P., and Walker, A.R. (2006). Light-induced expression of a MYB gene regulates anthocyanin biosynthesis in red apples. Plant Physiol. 142, 1216-1232. doi: 10.1104/pp.106.088104.

Tamura, K., Peterson, D., Peterson, N., Stecher, G., Nei, M., and Kumar, S. (2011). MEGA5: molecular evolutionary genetics analysis using maximum likelihood, evolutionary distance, and maximum parsimony methods. Mol. Biol. Evol. 28, 2731-2739. doi: 10.1093/molbev/msr121.

Voipio, I., and Autio, J. (1995). Responses of red-leaved lettuce to light intensity, UV-A radiation and root zone temperature. Acta Hortic. 399, 183-190. doi: https://doi.org/10.17660/ActaHortic.1995.399.21.

Winkel-Shirley, B. (2001). Flavonoid biosynthesis. A colorful model for genetics, biochemistry, cell biology, and biotechnology. Plant physiol. 126, 485-493. doi: https://doi.org/10.1104/pp.126.2.485.

$\mathrm{Xu}, \mathrm{W} .$, Dubos, C., and Lepiniec, L. (2015). Transcriptional control of flavonoid biosynthesis by MYB-bHLH-WDR complexes. Trends Plant Sci. 20, 176-185. doi: 10.1016/j.tplants.2014.12.001.

Yamagishi, M. (2016). A novel R2R3-MYB transcription factor regulates light-mediated floral and vegetative anthocyanin pigmentation patterns in Lilium regale.Mol.Breeding 36: 3. https://doi.org/10.1007/s11032-015-0426-y.

Zhang, F., Gonzalez, A., Zhao, M., Payne, C.T., and Lloyd, A. (2003). A network of redundant bHLH proteins functions in all TTG1-dependent pathways of Arabidopsis. Development 130, 4859-4869. doi: 10.1242/dev.00681.

Zhang, H.N., Li, W.C., Wang, H.C., Shi, S.Y., Shu, B., Liu, L.Q., Wei, Y.Z., and Xie, J.H. (2016b). Transcriptome Profiling of Light-Regulated Anthocyanin Biosynthesis in the Pericarp of Litchi. Front Plant Sci 7, 963. doi: 10.3389/fpls.2016.00963.

Zhang, Y., Cheng, Y., Ya, H., Xu, S., and Han, J. (2015). Transcriptome sequencing of purple petal spot region in tree peony reveals differentially expressed anthocyanin structural genes. Front. Plant Sci. 6, 964. doi: 10.3389/fpls.2015.00964.

Zhang, Y.Z., Xu, S.Z., Cheng, Y.W., Ya, H.Y., and Han, J.M. (2016a). Transcriptome analysis and anthocyanin-related genes in red leaf lettuce. Genet. Mol. Res. 15. doi: 10.4238/gmr.15017023.

Zoratti, L., Karppinen, K., Luengo Escobar, A., Haggman, H., and Jaakola, L. (2014). Light-controlled flavonoid biosynthesis in fruits. Front. Plant Sci. 5, 534. doi: 10.3389/fpls.2014.00534. 


\section{Figure 1}

Color difference of red leaf lettuce

(A) Plant was grown under $40 \mu \mathrm{mol} \mathrm{m} \mathrm{m}^{-2} \mathrm{~s}^{-1}$ light as the control. (B) Plant was irradiated under $100 \mu \mathrm{mol} \mathrm{m} \mathrm{m}^{-2} \mathrm{~s}^{-1}$ light after 3 days. (C) Anthocyanins content of red leaf lettuce under different light intensity. (D) Soluble sugar content of red leaf lettuce under different light intensity. Photo credit: Yanzhao Zhang. 

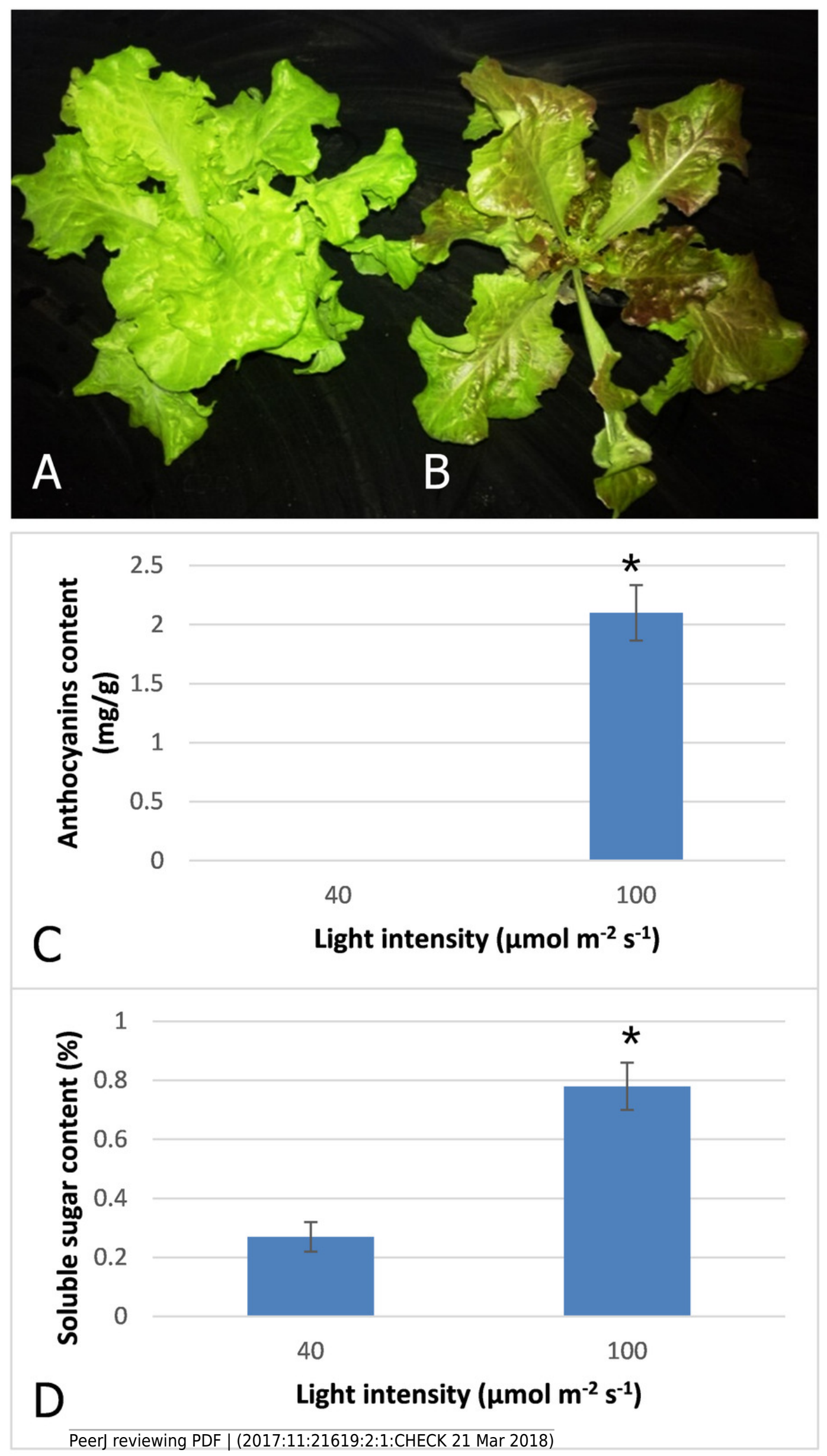
Figure 2

Volcano plot of differently expressed genes between red leaf lettuce under light intensity of 40 and $100 \mu \mathrm{mol} \mathrm{m} \mathrm{m}^{-2} \mathrm{~s}^{-1}$.

"FDR $\leq 0.01$ " and "log2 ratio $\geq 1$ " were used as thresholds to determine the different expressed genes (DEGs). Red points represent up-regulated DEGs, blue points represent down -regulated DEGs, and black points represent non-DEGs.

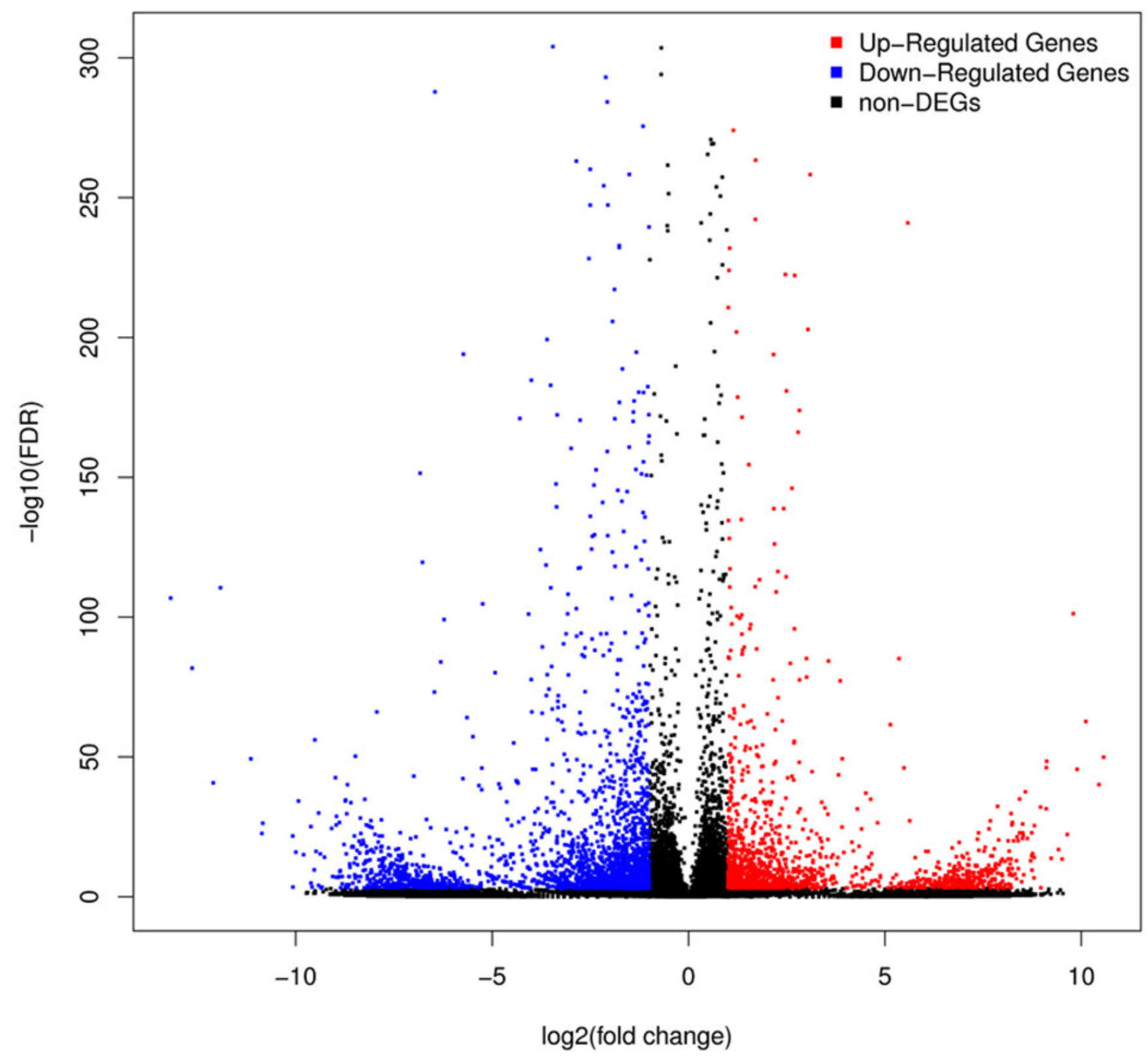




\section{Figure 3}

Pathway functional enrichment of DEGs.

$X$ axis represents enrichment factor. $Y$ axis represents pathway name. Coloring indicates $Q$ value (high: green, low: red),the lower $Q$ value indicates the more significant enrichment. Point size indicates DEG number (more: big, less: small).

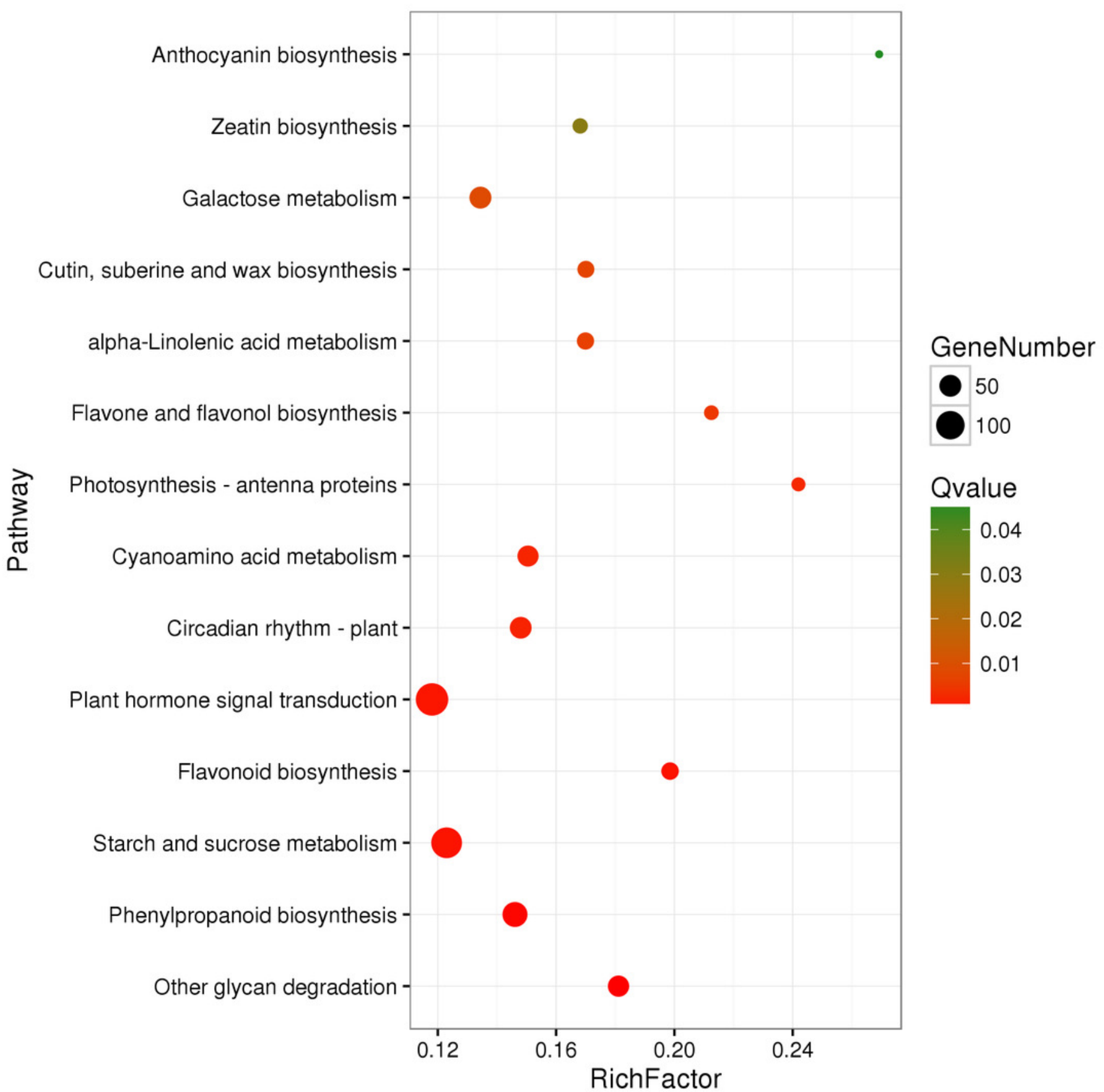




\section{Figure 4}

Phylogenetic analysis of anthocyanin biosynthesis transcription factors of red leaf lettuce.

(A) Phylogenetic tree of 5 MYBs with MYB transcription factors in other plants. The accession numbers are as follows: AtMYB12(NP_182268), AtMYB11(NP_191820), AtMYB111(NP_199744), AtTT2(Q9FJA2), AtWER(NP_196979), Ca A(CAE75745), GhMYB10(CAD87010), LeANT1(AAQ55181), MdMYB1(ABK58136), AtMYB0(NP_189430), MYB75(NP_176057), MYB90 (NP_176813), MYB113(NP_176811), MYB114(NP_176812), NtAN2 (NP_001306786), PhAN2(AAF66727), PhDPL(ADW94950), PhPHZ(ADW94951), Rosea1(ABB83826), Rosea2(ABB83827), VENOSA(ABB83828), VvMYBA1(AB242302), VvMYBA2(AB097924). (B) Phylogenetic tree of Unigene13011_All with bHLH proteins in others plants. The accession numbers are as follows: AmDELILA(AAA32663), AtMYC1(BAA11933), AtEGL1 (NP_176552), AtGL3(NP_001332705), PhJAF13(AAC39455), MdbHLH3(MdbHLH3), MdbHLH33(DQ266451), PhAN1(AAG25928), AtTT8(CAC14865), ZmB(CAA40544)! ZmLC(AAA33504), DvIVS(BAM8423 9), LcbHLH2(APP94123).
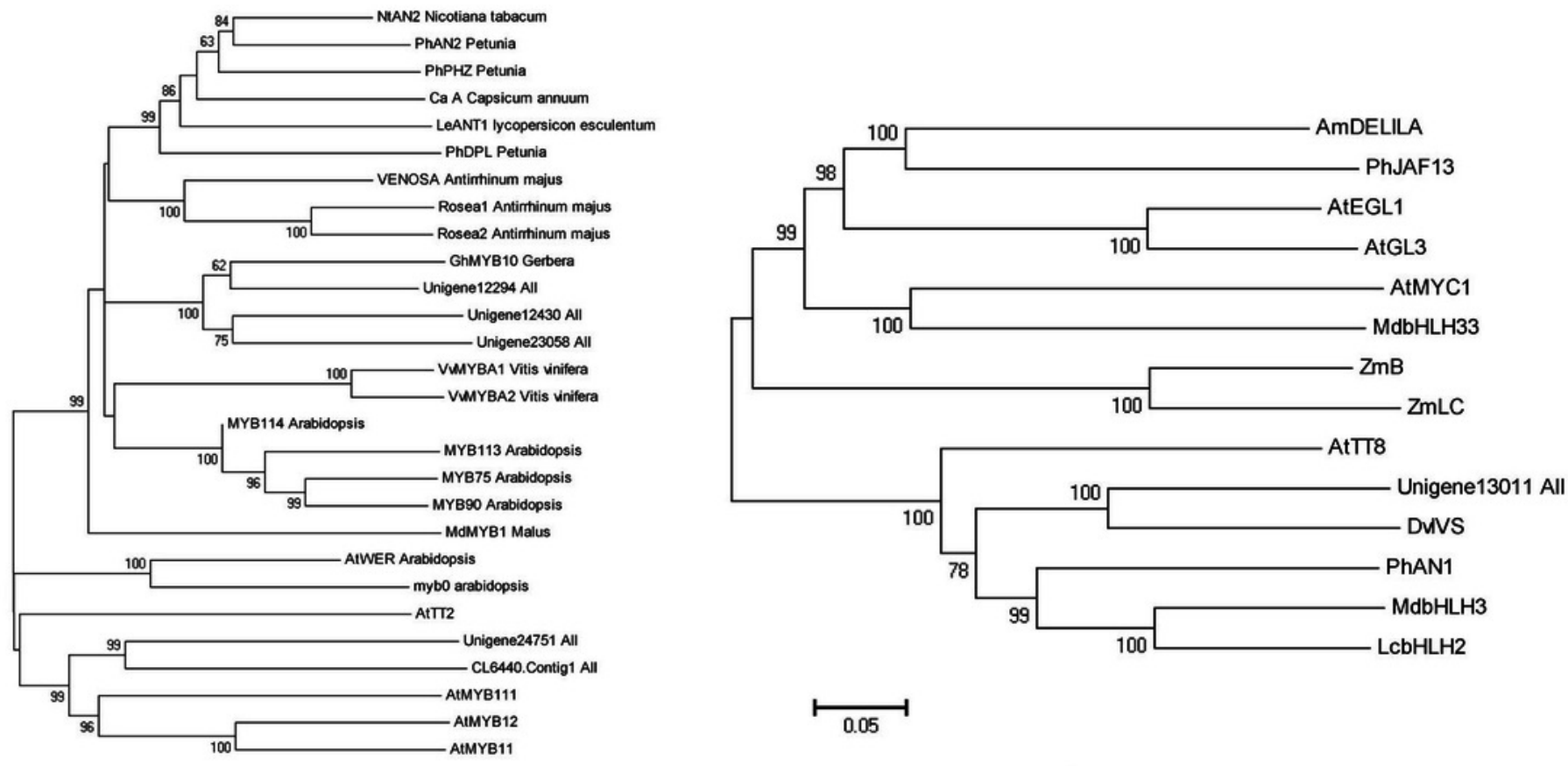
Figure 5

qRT-PCR validation of differentially expressed genes related to anthocyanin.

(A) to $(R)$ represent expression levels of different genes. $X$ axis represents light intensity $\left(\mu \mathrm{mol} \mathrm{m} \mathrm{m}^{-2} \mathrm{~s}^{-1}\right), \mathrm{Y}$ axis represents relative transcription level. All values are normalized relative to the abundance of actin gene. qRT-PCR analysis were performed in three biological and technical replicates per experiment. The bars represent means \pm SD from triplicate biological repeats.
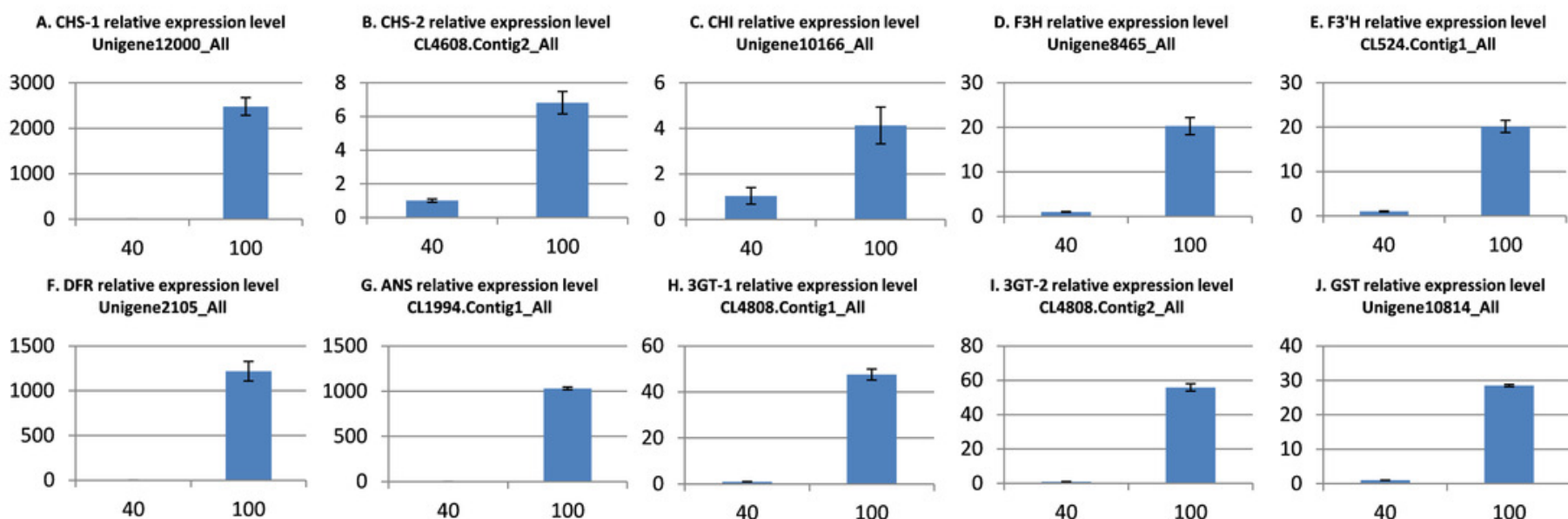
CL4808.Contig1_All

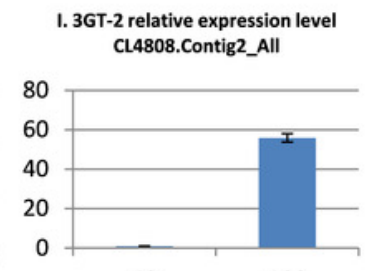

J. GST relative expression level Unigene10814_All

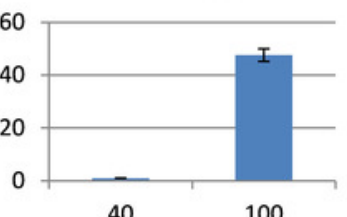

40

40

100

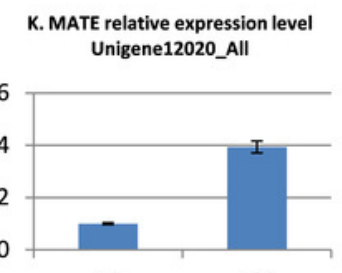

$40 \quad 100$

P. MYB-4 relative expression level Unigene24751_All

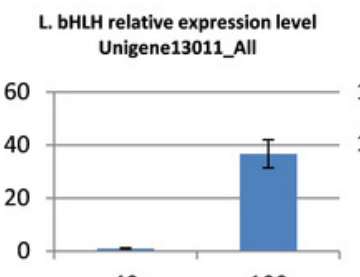

M. MYB-1 relative expression level Unigene12430_All

N. MYB-2 relative expression level Unigene12294_All

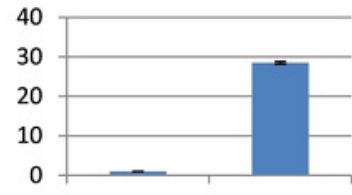

40

100

O. MYB-3 relative expression level Unigene23058_All

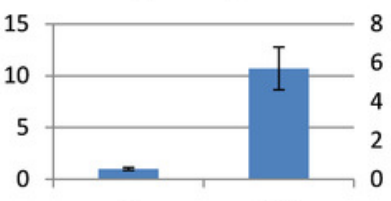

40

100
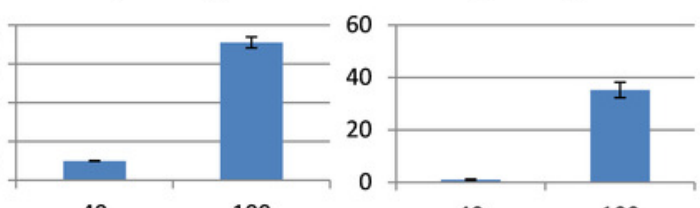

R. HY5 relative expression level Unigene19629_All

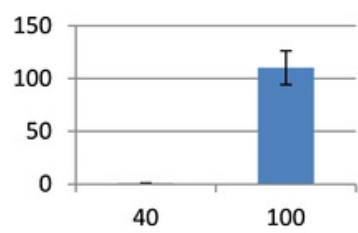

Q. MYB-5 relative expression level CL6440.Contig1_All

40

40
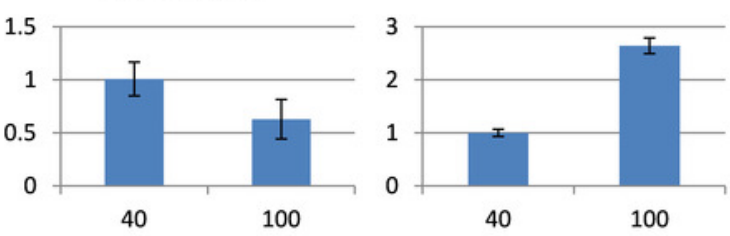
Table $\mathbf{1}$ (on next page)

Differentially expressed genes related with anthocyanin. 
1 Table 1. Differentially expressed genes related with anthocyanin

\begin{tabular}{|c|c|c|c|c|c|}
\hline Gene name & $\begin{array}{l}\text { low_light } \\
\text { FPKM }\end{array}$ & $\begin{array}{l}\text { high_light } \\
\text { FPKM }\end{array}$ & $\begin{array}{l}\text { log2FoldChange } \\
\text { (high/low light) }\end{array}$ & FDR & Up/Down \\
\hline \multicolumn{6}{|l|}{ LSCHS } \\
\hline Unigene12000_All & 1.68 & 858.26 & 9.0 & 0 & Up \\
\hline CL4608.Contig2_All & 11.36 & 97.28 & 3.1 & $5.33 e-259$ & Up \\
\hline \multicolumn{6}{|l|}{ LsCHI } \\
\hline Unigene10166_All & 10.6 & 68.72 & 2.70 & $1.43 e-96$ & Up \\
\hline \multicolumn{6}{|l|}{ LsF3H } \\
\hline Unigene8465_All & 14.21 & 408.44 & 4.85 & 0 & Up \\
\hline \multicolumn{6}{|l|}{ LsF3'H } \\
\hline CL524.Contig1_All & 9.08 & 193.8 & 4.42 & 0 & Up \\
\hline \multicolumn{6}{|l|}{ LsDFR } \\
\hline Unigene2105_All & 2.3 & 473 & 7.68 & 0 & Up \\
\hline \multicolumn{6}{|l|}{ LSANS } \\
\hline CL1994.Contig1_All & 4.25 & 269.69 & 5.99 & 0 & Up \\
\hline \multicolumn{6}{|l|}{ Ls3GT } \\
\hline CL4808.Contig1_All & 5.84 & 158.28 & 4.76 & 0 & Up \\
\hline CL4808.Contig2_All & 3.31 & 84.6 & 4.66 & 0 & Up \\
\hline \multicolumn{6}{|l|}{ LsGST } \\
\hline Unigene10814_All & 1.84 & 245.41 & 7.06 & 0 & Up \\
\hline \multicolumn{6}{|l|}{ LsMATE } \\
\hline Unigene12020_All & 8.42 & 18.14 & 1.11 & $9.25 e-15$ & Up \\
\hline \multicolumn{6}{|l|}{ LSMYB } \\
\hline Unigene12430_All & 2.21 & 8.30 & 1.91 & $4.36 \mathrm{e}-10$ & Up \\
\hline Unigene12294_All & 3.14 & 37.14 & 3.56 & $5.02 e-85$ & Up \\
\hline Unigene23058_All & 0.11 & 7.25 & 6.04 & $5.68 \mathrm{e}-17$ & Up \\
\hline Unigene24751_All & 0.56 & 22.95 & 5.36 & $7.05 e-86$ & Up \\
\hline CL6440.Contig1_All & 0.72 & 4.60 & 2.66 & $9.14 \mathrm{e}-11$ & Up \\
\hline \multicolumn{6}{|l|}{ LsbHLH } \\
\hline Unigene13011_All & 3.64 & 21.95 & 2.59 & $3.37 e-84$ & Up \\
\hline
\end{tabular}




\section{LsHY5}

Unigene19629_All

3.18

10.42

1.71

$3.15 \mathrm{e}-05$

Up

2 